\title{
Short communication: Genotyping and single nucleotide polymorphism analysis of bovine leukemia virus in Chinese dairy cattle
}

\author{
Y. Yang, ${ }^{1,2 *}$ S. Chu, ${ }^{3}$ S. Shang, ${ }^{1,2}$ Z. Yang, ${ }^{2,3}$ and C. Wang ${ }^{4}$ \\ ${ }^{1}$ Jiangsu Co-innovation Center for Prevention and Control of Important Animal Infectious Diseases and Zoonoses, College of Veterinary Medicine, \\ Yangzhou University, Yangzhou 225009, Jiangsu, China \\ ${ }^{2}$ International Corporation Laboratory of Agriculture and Agricultural Products Safety, Yangzhou University, Yangzhou 225009, Jiangsu, China \\ ${ }^{3}$ College of Animal Science and Technology, Yangzhou University, Yangzhou 225009, Jiangsu, China \\ ${ }^{4}$ Department of Pathobiology, College of Veterinary Medicine, Auburn University, Auburn, AL 36849
}

\section{ABSTRACT}

Bovine leukemia virus (BLV) causes enzootic leucosis in cattle and is classified into 10 genotypes with a worldwide distribution, except for several European countries, Australia, and New Zealand. Although BLV is widespread in Chinese cows with the positive rate of $49.1 \%$ at the individual level, very little is known about the BLV genotype in dairy cattle in China. To determine BLV genetic variability in cows in China, 112 BLV-positive samples from 5 cities in China were used for BLV molecular characterization in this study. Phylogenetic analysis using the neighbor-joining method on partial env sequence encoding gp 51 obtained from 5 Chinese cities and those available in GenBank $(\mathrm{n}=53$, representing BLV genotype 1-10) revealed the Chinese strains belonged to genotype 6 . Seven unique SNP were identified among Yancheng, Shanghai, and Bengbu strains out of the total 12 SNP identified in Chinese strains. The genotyping coupled with SNP analysis of BLV can serve as a useful molecular epidemiological tool for tracing the source of pathogens. This study highlights the importance of genetic analysis of geographically diverse BLV strains to understand BLV global genetic diversity.

Key words: bovine leukemia virus, genotype, single nucleotide polymorphism, Chinese dairy cattle

\section{Short Communication}

Bovine leukemia virus (BLV), which belongs to the family of Retroviridae and genus Deltaretrovirus, is genetically closely related to human T-cell leukemia virus-1. The BLV genome consists of 8,714 nucleotides including gag, pro, pol, env, R3, G4, tax, and rex genes, which are flanked by 2 identical long-terminal repeats.

Received August 22, 2018.

Accepted December 5, 2018.

*Corresponding author: yangyi@yzu.edu.cn
The env encodes a mature surface glycoprotein (gp51) and a transmembrane protein (gp30), which are primarily used for BLV genotyping. Presently, BLV are classified into 10 genotypes based on the env gene. Genotype 1 is the most widespread and has already been identified in many countries including the United States, Australia, Brazil, Uruguay, Paraguay, and Japan (Coulston et al., 1990; Camargos et al., 2002; Zhao and Buehring, 2007; Moratorio et al., 2010; Polat et al., 2016). Bovine leukemia virus genotypes 2 to 10 were regional epidemics, whereas genotype 6 was primarily described in South America (Brazil and Paraguay; Polat et al., 2016) and genotype 10 was recently described in Southeast Asia (Thailand and Myanmar; Lee et al., 2016; Polat et al., 2017a).

Our recent study showed that BLV is widespread in Chinese dairy cattle and the positive rate was $49.1 \%$ at the individual level (Yang et al., 2016a). Subsequently, Wang et al. (2018) investigated the molecular epidemiology and characterization of BLV in domestic yaks (Bos grunniens) on the Qinghai-Tibet Plateau, China, and identified 2 BLV genotypes (genotypes 6 and 10). However, to date, little information is available on the genotypes of BLV in Chinese dairy cattle. Thus, the aim of this study is to investigate the genotypes of BLV in Chinese dairy cattle.

During 2013 and 2014, 964 BLV fluorescence resonance energy transfer quantitative PCR (FRET-qPCR) positives were identified from 1,963 dairy blood samples from Yancheng (35/370), Shanghai (526/654), Yangzhou (206/343) Bengbu (9/108), Tianjin (188/282), Qiqihar (0/99), and Beijing (0/107; Yang et al., 2016a). Copy numbers of BLV in positive cows ranged from 20 copies/mL to $6,810,829$ copies/mL and those of above 17,717 copies/mL were enrolled in this study. In total, 112 genomic DNA samples of BLV-positive cattle were chosen from Yancheng $(\mathrm{n}=7)$, Shanghai $(\mathrm{n}=49)$, Yangzhou ( $\mathrm{n}=40)$, Bengbu $(\mathrm{n}=4)$, and Tianjin ( $\mathrm{n}$ $=12$ ). Regular PCR based on the BLV env gp51 gene were performed with a pair of primers described in our 


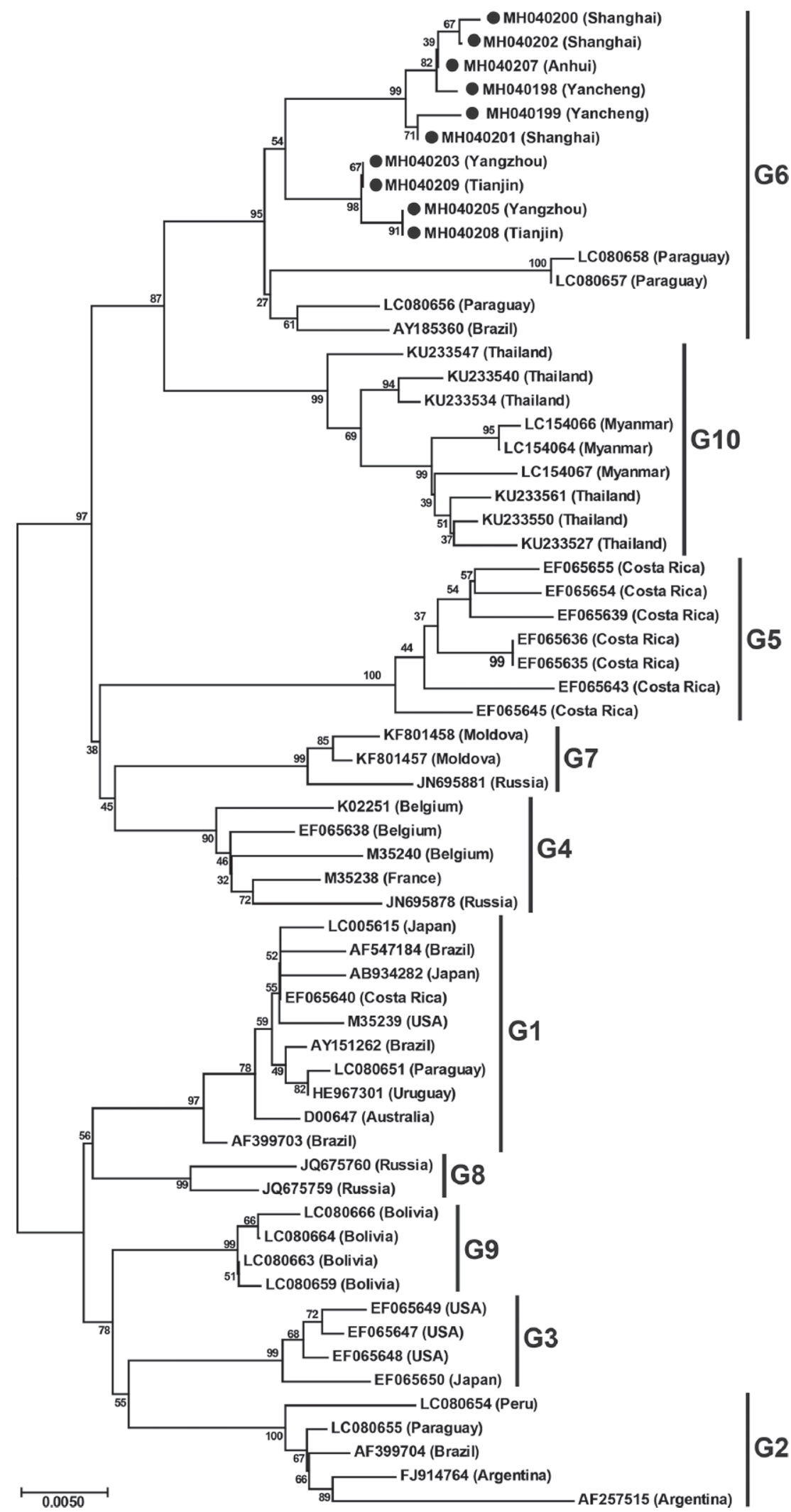

Figure 1. Neighbor-joining phylogenetic tree based on env gp51 sequences (805 bp) of bovine leukemia viruses from China and around the world. Strains identified in our study from China are identified with filled circles $(\bullet)$. Genotypes shown on the right are according to Yang et al. (2016b). The numbers at the branches show bootstrap support (1,000 replicates). The bar at the bottom of the figure denotes distance. 
previous study (Yang et al., 2016b). Amplicons were gel purified with the QIAquick Gel Extraction Kit and sequenced at the Genomic Sequencing Laboratory (GBI, Shanghai, China). Sequence data obtained in this study were assembled with DNASTAR Lasergen 15.2 (DNASTAR Inc., Madison, WI) and aligned using CLUSTAL W in MEGA 7.0 (MEGA, Pennsylvania State University, University Park) along with those of BLV strains found on GenBank from around the world. A neighbor-joining phylogenetic tree was constructed using the Tamura-Nei model (Rola-Euszczak et al., 2013; Polat et al., 2015) and the robustness of clusters was assessed by bootstrapping 1,000 replicates. Maximum-likelihood phylogenetic analysis was performed to confirm the results.

The sequences obtained in our study that were not identical to each other were submitted to GenBank with the GenBank accession numbers: MH040198MH040203, MH040205, and MH040207-MH040209. A neighbor-joining phylogenetic tree based on the env gene (805 bp, including the gp51 encoding region) sequences of the Chinese strains and 53 reference strains representing BLV genotypes 1 to 10 from 15 countries demonstrated that all the Chinese strains belonged to genotype 6 (Figure 1).

The maximum-likelihood analysis also showed that the Chinese strains clustered within genotype 6 . The mean distance of the $e n v$ nucleotides and the deduced AA were $0.015 \pm 0.003$ and $0.042 \pm 0.008$, respectively, for the 10 Chinese BLV strains identified in this study (Table 1). Compared with the BLV strains obtained from GenBank representing BLV genotypes 1 to 10, the Chinese strains had between $0.021 \pm 0.003$ (genotype 6) and $0.047 \pm 0.003$ (genotype 2) nucleotide distance. Similarly, the Chinese strains had between $0.058 \pm 0.011$ (genotype 6) and $0.108 \pm 0.019$ (genotype 2) deduced AA distance compared with these reference strains (Table 1). The distance of nucleotide and deduced AA indicated that the Chinese BLV strains were mostly similar to genotype 6 but distinct from genotype 10 .

To date, 10 BLV genotypes have been discovered around the world and among them BLV genotype 1 is the most widespread and has already been identified in Asia, North America, South America, and Oceania (Coulston et al., 1990; Camargos et al., 2002; Zhao and Buehring, 2007; Moratorio et al., 2010; Polat et al., 2016). Interestingly, genotype 1 has not been found in infected Chinese dairy cattle. Instead, genotype 6, mainly prevalent in South America (Polat et al., 2016), is the only genotype that has been identified in Chinese dairy cattle. Both Oceania and South America are the major exporters of dairy cattle to China. About 100 thousand dairy cattle were imported from Australia, New Zealand, Uruguay, and Chile to China. Among

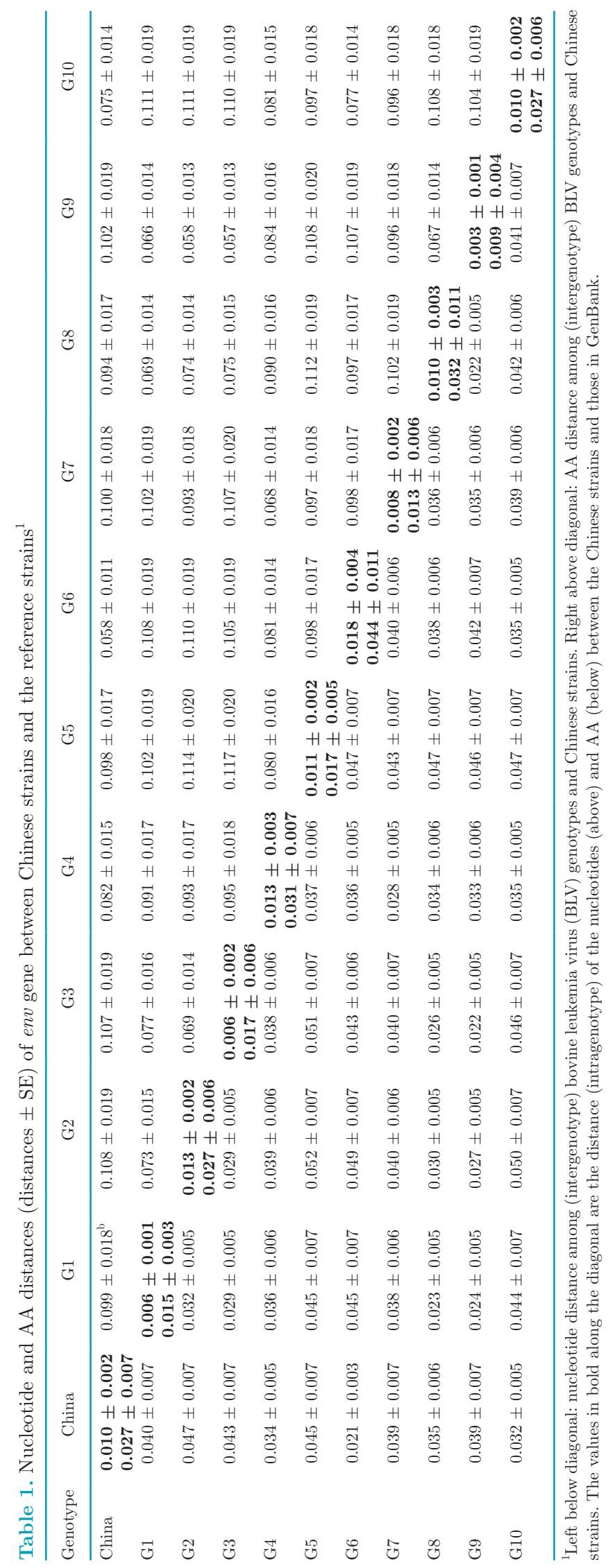

Journal of Dairy Science Vol. 102 No. 4, 2019 

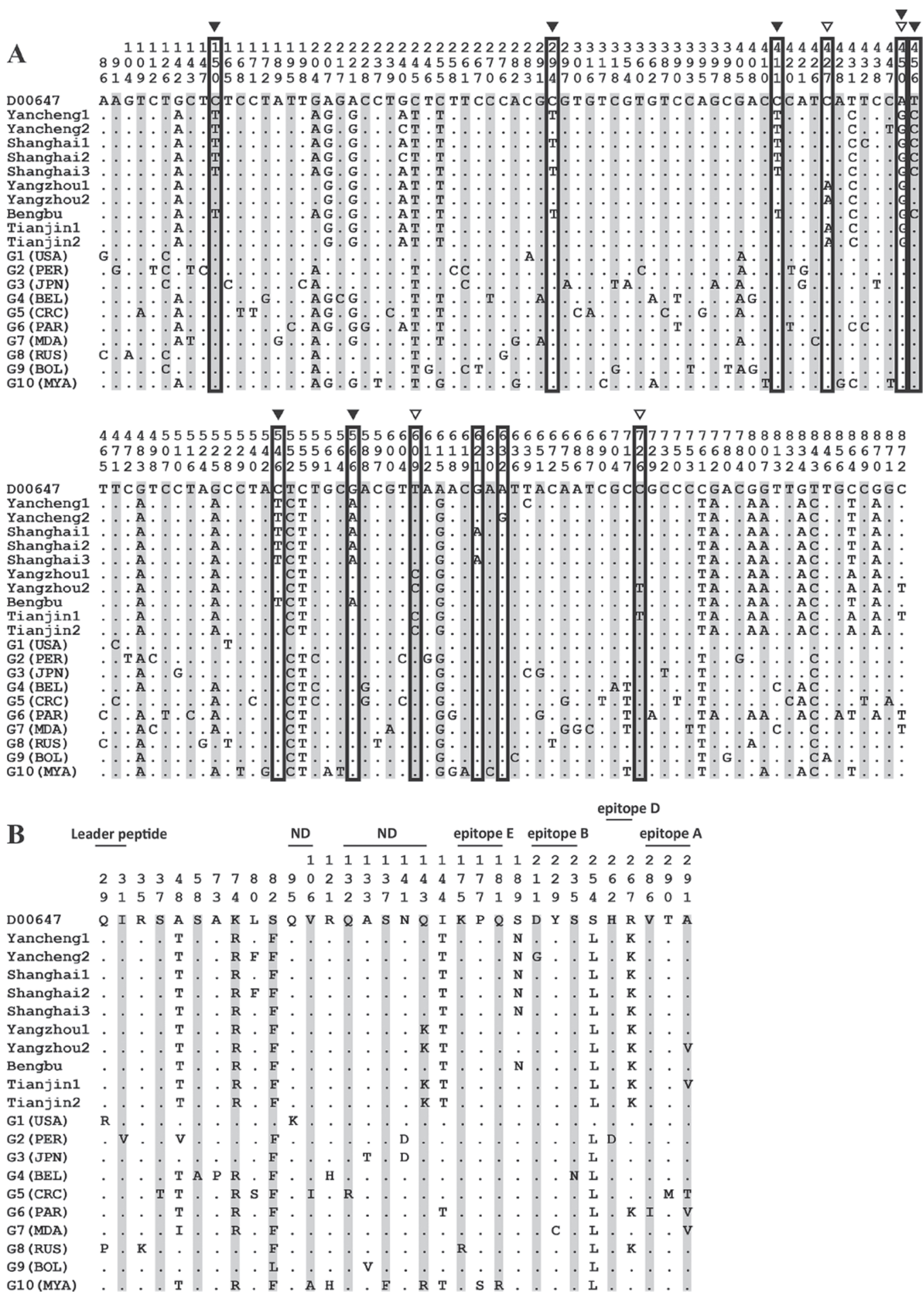

Figure 2. Alignment of partial bovine leukemia virus (BLV) env (805 bp) nucleotide (A) and deduced AA (B) sequences between Chinese and reference strains. Numbers above the sequences are nucleotide number and AA residue number indicated by the env gene of D00647. The countries of the strains are marked with abbreviations in parentheses to the right of the GenBank accession numbers. Dots indicate nucleotides or AA identical to the reference sequence. The square frames indicate the strains that have unique SNP. The mark above the square frames indicate identical SNP for Yancheng/Shanghai/Bengbu strains $(\mathbf{\nabla})$ and Yangzhou/Tianjin strains $(\nabla)$. Labeled lines indicate the position of identified glycoproteins, epitopes, and neutralization domains. The BLV reference strains from GenBank have accession numbers M35239 (G1), LC080654 (G2), EF065650 (G3), M35240 (G4), EF065655 (G5), LC080658 (G6), KF801458 (G7), JQ675760 (G8), LC080666 (G9), and LC154067 (G10). The 10 Chinese strains from this study have the accession numbers MH040198-MH040203, MH040205, and MH040207-MH040209. USA = United States; PER = Peru; JPN = Japan; BEL = Belgium; CRC = Costa Rica; PAR = Paraguay; MDA = Moldova; RUS $=$ Russia; BOL $=$ Bolivia; MYA = Myanmar. 
them, two-thirds of the cows were imported from Oceania and the remaining one-third from South America. Nationwide BLV eradication and control programs were introduced in Australia and New Zealand in 1983 and 1996, respectively, and $99.7 \%$ of Australian dairy herds were declared free of BLV in December 2013, whereas those in New Zealand have been free of BLV since 2008 (Polat et al., 2017b). This may partly explain why we failed to find genotype 1 , which was the most widespread BLV genotype in Oceania decades ago.

In this study, based on the nucleotide and deduced AA substitutions, the alignment of Chinese strains from this study and representative BLV strains (genotypes 1-10) identified a total of 12 unique SNP (the substitutions are different from all of the reference strains). Nine of these 12 SNP were synonymous SNP (C150T, C294T, C411T, A450G, T456C, C546T, T609C, G621A, and $\mathrm{C} 726 \mathrm{~T}$ ) and the remaining 3 were nonsynonymous SNP (C427A, G566A, and A632G; Figure 2A and 2B). In total, 7 identical SNP (nucleotides 150, 294, 411, 450, 456,546 , and 566) were found among all or partial strains from Yancheng, Shanghai, and Bengbu, whereas 4 identical SNP (nucleotides 427, 450, 609, and 726) were present in all or partial strains from Yangzhou and Tianjin, among which only one identical SNP (nucleotide 450) was shared between Yancheng/Shanghai/Bengbu strains and Yangzhou/Tianjin strains (Figure 2A). The background information showed that the cattle in Yancheng, Shanghai, and Bengbu were all introduced from Chile before 2010, whereas the cattle in Yangzhou and Tianjin were purchased domestically. Based on the SNP analysis of the gp51 gene, infected cows with the same source have more identical unique SNP for BLV than the cows with different sources.

Bovine leukemia virus is widespread among Chinese dairy herds, and BLV infection is associated with reduced milk yield and increased SCS. This study for the first time identified the genotype of BLV in infected Chinese dairy cattle. Further studies are needed to determine the possible association between BLV genotypes and the pathogenicity of this virus. Moreover, the BLV gp51 gene SNP analysis can probably serve as a useful molecular epidemiological tool, especially for tracing the source of pathogen in the event of a BLV outbreak.

In conclusion, this study revealed that at least BLV genotype 6 is present in Chinese dairy cattle and quarantine is needed when introducing cows from the epidemic focus.

\section{ACKNOWLEDGMENTS}

This project was supported by the National Natural Science Foundation of China (31472067, 31872324), Jiangsu Modern Agricultural Industry Technology System (Dairy Cow), and the Priority Academic Program Development of Jiangsu Higher Education Institutions (China).

\section{REFERENCES}

Camargos, M. F., D. Stancek, M. A. Rocha, L. M. Lessa, J. K. Reis, and R. C. Leite. 2002. Partial sequencing of env gene of bovine leukaemia virus from Brazilian samples and phylogenetic analysis. J. Vet. Med. B Infect. Dis. Vet. Public Health 49:325-331.

Coulston, J., H. Naif, R. Brandon, S. Kumar, S. Khan, R. C. Daniel, and M. F. Lavin. 1990. Molecular cloning and sequencing of an Australian isolate of proviral bovine leukaemia virus DNA: Comparison with other isolates. J. Gen. Virol. 71:1737-1746.

Lee, E., E. J. Kim, J. Ratthanophart, R. Vitoonpong, B. H. Kim, I. S. Cho, J. Y. Song, K. K. Lee, and Y. K. Shin. 2016. Molecular epidemiological and serological studies of bovine leukemia virus (BLV) infection in Thailand cattle. Infect. Genet. Evol. 41:245-254.

Moratorio, G., G. Obal, A. Dubra, A. Correa, S. Bianchi, A. Buschiazzo, J. Cristina, and O. Pritsch. 2010. Phylogenetic analysis of bovine leukemia viruses isolated in South America reveals diversification in seven distinct genotypes. Arch. Virol. 155:481-489.

Polat, M., H. H. Moe, T. Shimogiri, K. K. Moe, S. N. Takeshima, and Y. Aida. 2017a. The molecular epidemiological study of bovine leukemia virus infection in Myanmar cattle. Arch. Virol. 162:425-437.

Polat, M., A. Ohno, S. N. Takeshima, J. Kim, M. Kikuya, Y. Matsumoto, C. N. Mingala, M. Onuma, and Y. Aida. 2015. Detection and molecular characterization of bovine leukemia virus in Philippine cattle. Arch. Virol. 160:285-296.

Polat, M., S. N. Takeshima, and Y. Aida. 2017b. Epidemiology and genetic diversity of bovine leukemia virus. Virol. J. 14:209.

Polat, M., S. N. Takeshima, K. Hosomichi, J. Kim, T. Miyasaka, K. Yamada, M. Arainga, T. Murakami, Y. Matsumoto, V. de la Barra Diaz, C. J. Panei, E. T. Gonzalez, M. Kanemaki, M. Onuma, G. Giovambattista, and Y. Aida. 2016. A new genotype of bovine leukemia virus in South America identified by NGS-based whole genome sequencing and molecular evolutionary genetic analysis. Retrovirology 13:4.

Rola-Łuszczak, M., A. Pluta, M. Olech, I. Donnik, M. Petropavlovskiy, A. Gerilovych, I. Vinogradova, B. Choudhury, and J. Kuźmak. 2013. The molecular characterization of bovine leukaemia virus isolates from Eastern Europe and Siberia and its impact on phylogeny. PLoS One 8:e58705.

Wang, M., Y. Wang, A. R. Baloch, Y. Pan, F. Xu, L. Tian, and Q. Zeng. 2018. Molecular epidemiology and characterization of bovine leukemia virus in domestic yaks (Bos grunniens) on the QinghaiTibet Plateau, China. Arch. Virol. 163:659-670.

Yang, Y., W. Fan, Y. Mao, Z. Yang, G. Lu, R. Zhang, H. Zhang, C. Szeto, and C. Wang. 2016a. Bovine leukemia virus infection in cattle of China: Association with reduced milk production and increased somatic cell score. J. Dairy Sci. 99:3688-3697.

Yang, Y., P. J. Kelly, J. Bai, R. Zhang, and C. Wang. 2016b. First molecular characterization of bovine leukemia virus infections in the Caribbean. PLoS One 11:e0168379.

Zhao, X., and G. C. Buehring. 2007. Natural genetic variations in bovine leukemia virus envelope gene: Possible effects of selection and escape. Virology 366:150-165. 